\title{
émulations
}

\section{Le rôle de l'espace public dans le développement de la vie nocturne (Montpellier-Lisbonne)}

\author{
Emanuele Giordano et Pedro Gomes
}

Émulations - Revue de sciences sociales

2020, n 33, "La nuit urbaine. Un espace-temps complexe entre opportunités et inégalités ».

\section{Article disponible à l'adresse suivante}

https://ojs.uclouvain.be/index.php/emulations/article/view/giordano_gomes

\section{Pour citer cet article}

Emanuele Giordano et Pedro Gomes, « Le rôle de l'espace public dans le développement de la vie nocturne (Montpellier-Lisbonne) », Émulations, n 33, Mise en ligne le 15 juin 2020.

DOI : 10.14428/emulations.033.04

Distribution électronique : Université catholique de Louvain (Belgique) : ojs.uclouvain.be

(C) Cet article est mis à disposition selon les termes de la Licence Creative Commons Attribution, Pas d'Utilisation Commerciale 4.0 International. http://creativecommons.org/licenses/by-nc/4.0/

Éditeur : Émulations - Revue de sciences sociales / Presses universitaires de Louvain https://ojs.uclouvain.be/index.php/emulations

ISSN électronique : 1784-5734

PUL PRESSES

UNIVERSITAIRES

DE LOUVAIN 


\title{
Le rôle de l'espace public dans le développement de la vie nocturne (Montpellier-Lisbonne)
}

\author{
Emanuele Giordano ${ }^{1}$ et Pedro Gomes ${ }^{2}$
}

\begin{abstract}
[Résumé] L'évolution des loisirs nocturnes a été explorée dans sa relation avec les processus de déréglementation des activités commerciales et avec des évolutions des modes de vie. Cependant, le rôle joué par les transformations dans la production de la matérialité de la ville, et en particulier de l'espace public, reste relativement peu interrogé. À partir des cas de Montpellier et de Lisbonne, cet article montre que les conflictualités associées au développement des loisirs nocturnes relèvent de plusieurs impensés et ambiguïtés dans la prise en compte des usages et de leurs temporalités dans la production urbaine, notamment des espaces publics. Ce faisant, l'article montre comment la nuit peut être un espace-temps privilégié pour analyser certaines des ambiguïtés et contradictions qui caractérisent les politiques de revitalisation des centres historiques des villes européennes.
\end{abstract}

Mots-clés : espace public, nuit, urbanisme, usages.

\section{The role of public space in the development of nightlife (Montpellier-Lisbonne)}

[Abstract] The development of the night-time economy has been linked to the changing regulation of commercial activities, as well as broader social and cultural transformations. However, the role played by urban planning and design remains scarcely addressed. Based on the cases of Montpellier and Lisbon, this article shows how conflicts associated with the development of nocturnal leisure activities arise from a lack of consideration for urban practices and their temporality in the production and management of public spaces. The article further shows how the night can be a privileged space-time to analyze some of the ambiguities and contradictions that characterize the policies of revitalization of the historic centers of European cities.

Keywords: public space, night, urban planning, urban practices.

\section{Introduction}

Devant l'importance croissante des loisirs nocturnes en contexte urbain du point de vue économique, social et culturel, la plupart des travaux académiques ont insisté sur les processus de déréglementation et de développement de l'industrie de l'alcool et des loisirs nocturnes dans plusieurs pays, souvent liés à des visions d’inspiration néolibérale (Bianchini, 1995). Plusieurs auteurs ont alors interrogé le rôle des processus de déréglementation des licences d'alcool et des horaires d'ouverture, d'une part (Wolifson, Drozdzewski, 2017), et d'autre part, l'augmentation de la demande liée aux évolutions

\footnotetext{
${ }^{1}$ Université de Toulon, laboratoire Babel EA 2649, France. Ce travail est soutenu par l'Agence nationale de la recherche (ANR), projet ANR-18-CE41-0005. URL : https://anr.fr/Projet-ANR-18CE41-0005.

2 Université Paris-Est, Lab'Urba EA 3482, France. La recherche présentée ici a été financée par la Fundação para a Ciência e Tecnologia (FCT, référence SFRH/BD/78852/2011).
} 
culturelles et sociales (Elias, Dunning, 1986). Cependant, le rôle joué par les transformations liées aux loisirs nocturnes dans la production et l'organisation de la matérialité de la ville contemporaine, et en particulier de l'espace public, reste relativement peu interrogé. À partir de travaux de recherche développés par les auteurs à Montpellier et Lisbonne, cet article se propose de répondre à un double objectif. En utilisant la nuit à la fois comme objet et terrain d'étude (Nofre, Eldridge, 2018), l'article explore la façon dont les conflictualités associées au développement des loisirs nocturnes relèvent, au moins partiellement, de plusieurs impensés et ambiguïtés dans la prise en compte des usages et de leurs temporalités dans la planification urbaine et dans la production et la régulation des espaces publics. En même temps, il montre comment la nuit peut être un espace-temps privilégié pour analyser certaines des ambiguïtés et contradictions qui caractérisent les politiques de revitalisation des centres historiques des villes européennes. Pour ce faire, cet article propose une mise en perspective a posteriori (Geoffray et al., 2012) de deux recherches menées avec deux entrées distinctes : l'une portant sur les pratiques festives nocturnes (à Montpellier, France), l'autre sur la production des espaces publics (à Lisbonne, Portugal). Ces deux entrées se complètent, l'une partant des pratiques urbaines pour ensuite questionner leur régulation par l'action publique, l'autre portant sur la prise en compte des usages dans la conception et l'aménagement de l'espace public. Le regard croisé sur ces deux villes est par ailleurs permis du fait de certains éléments communs aux deux villes : situées en Europe du Sud, elles accueillent une forte population étudiante ${ }^{3}$ et sont le lieu de politiques urbaines qui, depuis une trentaine d'années, visent à développer l'attractivité résidentielle et touristique (Camarinhas, 2011 ; Volle et al., 2010).

L'enquête menée à Montpellier entre janvier 2016 et décembre 2018 est fondée sur plusieurs techniques d'enquête. Tout d'abord, une analyse de nombreux documents de planification, de la presse locale et des arrêtés municipaux et de la préfecture. Ensuite, des observations flottantes (Pétonnet, 1982) menées entre $18 \mathrm{~h}$ et $5 \mathrm{~h}$ ont nourri une première analyse des pratiques nocturnes et du paysage urbain de l'Écusson (centre historique de la ville). Ces séances d'observation ont été complétées par sept entretiens semi-directifs avec des techniciens municipaux (directeurs et chargés de projet ou de mission) dans trois services (police municipale, services municipaux d'hygiène et de santé, d'urbanisme et occupation du domaine public), douze entretiens avec des résidents et cinq entretiens avec des acteurs actifs dans le quartier enquêté (un agent immobilier, deux gérants d'établissement, deux intervenants d'association de défense du voisinage du centre-ville). Enfin, un questionnaire a été distribué en ligne dans le

\footnotetext{
${ }^{3}$ Montpellier a une population de 281613 habitants et on estime à près de 71000 le nombre d'étudiants présents dans les deux universités montpelliéraines et les écoles supérieures (Ville de Montpellier. En ligne, consulté le 11 janvier 2020. URL : https://www.montpellier.fr/2754-universites.htm). En 2019, 120696 étudiants étaient inscrits dans les institutions d'enseignement supérieur de la commune de Lisbonne, dont la population résidente s'élève à 506654 habitants (données DGEEC/MEd - MCTES, Pordata et INE, Pordata. En ligne, consulté le 8 janvier 2020. URL : www.pordata.pt).
} 
premier semestre 2018 à des étudiants portant sur leurs pratiques nocturnes. Au total, 425 réponses ont été collectées.

À Lisbonne, l'enquête menée d'août 2012 à juillet 2015 comprend trente-six entretiens semi-directifs auprès d'acteurs locaux et l'analyse de nombreux documents de planification, ainsi que de la presse locale, source principalement utilisée dans cet articlé⿱

\section{Le développement des loisirs nocturnes au regard des pratiques d'urbanisme convivial}

La notion de Night-Time Economy (NTE) fait référence aux politiques de développement des activités festives nocturnes pour revitaliser les centres urbains en déclin. Initialement développée en Angleterre dans les années 1990, cette approche est aujourd'hui un phénomène global consistant en des politiques de développement des loisirs nocturnes avec l'objectif de dynamiser l'économie urbaine et de se positionner dans un contexte de compétitivité accrue entre les villes (van Liempt et al., 2015).

La plupart des chercheurs sont aujourd'hui d'accord (Shaw, 2015) pour placer la naissance de la NTE dans le contexte de l'émergence du paradigme néolibéral et d'entrepreneurialisation des politiques urbaines (Harvey, 1989). Elle était vue initialement comme une opportunité d'augmenter la rentabilité d'un temps traditionnellement jugé improductif (Bianchini, 1995), tout en permettant de civiliser les comportements selon la supposition qu'un centre-ville plus fréquenté serait également un centre-ville plus sûr, attirant à son tour un éventail plus large de personnes (Roberts, Eldridge, 2009). Cependant, ces promesses ne se sont pas réalisées dans le cas de plusieurs villes britanniques, où les industries de l'alcool et des loisirs ont été les mieux placées pour saisir les opportunités produites par la déréglementation. Ainsi, le développement de la NTE a été de plus en plus associé à l'augmentation de l'ivresse, des violences et des nuisances sonores (Hadfield et al., 2001).

Dans les décennies suivantes, cette stratégie a circulé à l'échelle globale. Dans un contexte caractérisé par une compétition interurbaine accrue et une " pression globale " pour stimuler le tourisme, plusieurs pays européens (Suède, Danemark, Italie) et l'Australie ont adopté des stratégies de déréglementation (Roberts, Eldridge, 2009 ; Wolifson, Drozdzewski, 2017). Aujourd'hui, la plupart des recherches soulignent les relations qui existent entre le développement des industries de l'alcool et des loisirs nocturnes et les processus de déréglementation d’inspiration néolibérale. Cependant, le développement de la NTE intéresse aussi des pays qui n'ont pas connu ce processus de déréglementation, comme la France (Nofre et al., 2018).

En effet, en France, la nuit est très peu considérée comme une source d'attractivité et de revenus (Comelli, 2016), tandis que la déréglementation du système de licence d'alcool français reste limitée au regard de la plupart des pays européens (Roberts et al., 2006). Cependant, les conflits autour d'usages nocturnes contradictoires n'y sont pas pour autant moins présents (Guérin, 2017 ; Walker, 2018). Dans ce cadre, plusieurs

\footnotetext{
${ }^{4}$ Pour plus de détails sur le cas de Lisbonne, voir Gomes (2017).
} 
travaux ont souligné la croissante importance économique et sociale des activités festives dans le processus de construction de l'identité individuelle (Chatterton, Hollands, 2003 ; voir l'article de Defrance dans ce numéro).

À l'inverse, le rôle joué dans cette dynamique par les transformations dans la production de la ville contemporaine, et en particulier de l'espace public, reste relativement peu interrogé. Or, les espaces publics urbains sont massivement réinvestis par une nouvelle génération de politiques urbaines entrepreneuriales (Fleury, 2007). Pour des villes désormais insérées dans le cadre d'un marché concurrentiel, l'espace public devient à la fois un élément structurant de l'identité urbaine, de son image de marque et un outil de recomposition du territoire. Il incarne un « urbanisme de l'offre », dans lequel les aménagements sont conçus pour accueillir, attirer et inciter des pratiques associées à un style de vie qui correspond aux publics privilégiés par les politiques en raison de leur capital économique et/ou social (Gomes, 2017)5. La libération de la place pour les piétons, la mise en place de mobilier pour des usages stationnaires, notamment des terrasses, participent ainsi au développement d'un urbanisme convivial ${ }^{6}$, souvent lié à la volonté des aménageurs et des élus de déclencher des processus de gentrification et/ ou de touristification (Rousseau, 2009).

Or, si cette transformation de la fabrique de l'espace public est envisagée comme un moyen pour modifier les usages spatiaux, elle a aussi souvent des impacts temporels, notamment une augmentation des conflits entre sortants (les individus engagés dans des pratiques de loisirs nocturnes dans l'espace public ou dans des établissements voués à cet effet) et résidents (Nofre, Eldridge, 2018). Dans cet article, nous montrerons que l'urbanisme convivial des centres-villes de Montpellier et de Lisbonne articule la promotion de la mobilité piétonne (à travers la piétonnisation, notamment) à l'encouragement d'usages stationnaires via l'introduction de mobilier urbain et la réhabilitation de commerces en pied d'immeuble. Ces solutions favorisent le développement de pratiques de loisirs nocturnes dans l'espace public, générateurs de conflits entre sortants et résidents.

\section{Les loisirs nocturnes, impensé de l'urbanisme convivial}

Les opérations de piétonnisation et de revitalisation des quartiers centraux s’inscrivent dans les politiques d'attractivité résidentielle, commerciale, touristique et, plus généralement, économique (Brennac et al., 2013). Si ces transformations socioéconomiques sont souhaitées par les élus et les concepteurs, ces opérations produisent aussi des transformations temporelles généralement moins maîtrisées (Mallet, 2013), dont des

\footnotetext{
${ }^{5}$ C'est le cas dans les processus étudiés dans cet article. Néanmoins, les pratiques d'urbanisme convivial peuvent également se destiner à d'autres publics et se décliner dans des contextes non centraux.

${ }^{6}$ L'urbanisme convivial désigne un ensemble de pratiques de gestion et de conception qui promeuvent explicitement la convivialité urbaine : l'interaction entre individus et entre individus et l'espace (Gomes, 2020). Souvent efficaces pour augmenter l'usage des espaces publics, ces pratiques sont fréquemment génératrices d'inégalités d'accès entre différents groupes sociaux.
} 
conflits produits par la difficile cohabitation entre sortants et riverains (Mallet, Burger, 2016). Les opérations entraînent souvent une spécialisation de l'offre commerciale en bars et restaurants (Özdemir, Selçuk, 2017) et des ambiances qui favorisent des usages conviviaux et prolongés des espaces publics pendant la nuit (Guérin, 2017).

Les cas de l'Écusson et du Cais do Sodré illustrent ces dynamiques. Situés respectivement dans les centres-villes de Montpellier et de Lisbonne, ces quartiers ont été historiquement caractérisés par un cadre bâti dégradé et une forte présence des classes populaires (Ferras, 1978 ; Nofre et al., 2018). À partir des années 2000, ils subissent d’importantes opérations de réhabilitation. À Montpellier, la municipalité lance en 2003 le programme Grand Cœur pour augmenter l'attractivité résidentielle du quartier via la réhabilitation du parc de logements, relancer l'attractivité commerciale du quartier et développer le tourisme ${ }^{7}$. À Lisbonne, ville confrontée à la persistance du déclin démographique et de l'emploi et à la dégradation du bâti, les objectifs sont similaires8 . Le centre-ville concentre plusieurs initiatives municipales de développement de son attractivité, renforçant les dynamiques existantes de "retour au centre » (Malheiros et al., 2012).

Dans les deux cas, la piétonnisation et/ou le réaménagement des espaces publics est un élément-clé des opérations. À l'Écusson, sur initiative de la mairie, plusieurs espaces publics sont réaménagés et le quartier est piétonnisé (Volle et al., 2010). À Lisbonne, les réaménagements d'espaces publics le long du front du Tage se succèdent depuis une dizaine d'années (Santos, 2019). La Rua Nova do Carvalho, dans le Cais do Sodré, est piétonnisée et peinte en rose en 2011 à l'initiative de l'association de commerçants, suite à l'arrivée d'un promoteur spécialisé dans la reconversion de friches. Son succès, attirant des milliers de personnes le week-end ${ }^{9}$, justifie un concours pour un réaménagement plus pérenne pour conforter de nouveaux usages diurnes (terrasses, promenade, galerie d'art en plein air) dans cette rue jusqu'alors fréquentée surtout pour ses boîtes de nuit. La nouvelle rue Rose, comme la Rua Nova do Carvalho est désormais désignée dans plusieurs supports de communication, est inaugurée en septembre 2013.

Ces types d'actions ont été souvent associées à des processus de gentrification (Clerval, Fleury, 2009 ; Rousseau, 2009). À Montpellier, la réhabilitation de l'Écusson contribue à un processus associant l'arrivée d'une population de cadres à une hausse des prix de l'immobilier (Giband, 2017). En 2018, le prix médian des appartements dans le centre-ville de Montpellier est de $3375 € / \mathrm{m}^{2}$ contre $2629 € / \mathrm{m}^{2}$ pour la ville ${ }^{10}$. La création de la rue Rose à Lisbonne, quant à elle, coïncide avec une montée des prix de

\footnotetext{
7 Ville de Montpellier (2017), Projet d'aménagement et de développement durable. En ligne, consulté le 29 septembre 2019. URL : https://www.montpellier.fr/380-le-plan-local-d-urbanisme-de-montpellier.htm\#toced_headerH2_2.

${ }^{8}$ Câmara Municipal de Lisboa (2011), Revisão do PDM. Relatório da Proposta de Plano, Lisbonne, Câmara Municipal de Lisboa.

${ }^{9}$ Cerejo J. A., «Câmara de Lisboa inaugura "Rua Rosa" com apoio de marca de vodka », Público, 31 août 2013. En ligne, consulté le 8 janvier 2020. URL : http://www.publico.pt/local-lisboa/jornal/ camara-de-lisboa-inaugura-rua-rosa-com-apoio-de-marca-de-vodka-27028929.

10 Observatoire départemental de l'Habitat 34 (ODH) (2019), Recul de l'accession à la propriété dans
} 
l'immobilier en centre-ville, fruit d'une nouvelle attractivité résidentielle mais surtout touristique (Lestegàs, 2019). À l'échelle de la rue, le réaménagement acte une montée en gamme de l'offre commerciale (offre à des prix plus élevés et qui cible des publics plus aisés, notamment des bars et restaurants) (Nofre, 2013).

Les interventions sur l'espace public ont aussi favorisé une spécialisation commerciale autour des loisirs nocturnes. Si cette spécialisation est cohérente avec les objectifs d'attractivité, elle a aussi produit une transformation des rythmes spatiotemporels. À Montpellier, elle a renforcé la vocation festive du quartier avec une augmentation importante du nombre d'établissements, qui perdure, voire s'amplifie :

Il y a une multiplication des lieux de nuit : il y a 6 ans, il y avait 500 établissements dans l'Écusson, aujourd'hui c'est 600 établissements ${ }^{11}$.

Suite à la piétonnisation, le nombre de demandes d'occupation du domaine public dans le centre-ville de Montpellier a beaucoup augmenté. Aujourd'hui, il y a environ 600 terrasses à Montpellier et environ 400 sont dans l'Écusson ${ }^{12}$.

On en a discuté avec les commerçants et ils sont d'accord. Ils nous disent qu'ils risquent de fermer leurs commerces qui seront remplacés par des bars ${ }^{13}$.

Aujourd'hui, l'Écusson représente le seul pôle festif nocturne de la ville, comme le confirment les résultats du questionnaire. Il est le quartier préféré pour sortir la nuit pour $97 \%$ des répondants.

Avant la piétonnisation de la Rua Nova do Carvalho, les usages festifs du Cais do Sodré se déroulaient surtout à l'intérieur des établissements (Nofre, 2013). Leur fréquentation avait d'ailleurs beaucoup augmenté en 2008, suite à la réduction des horaires de fonctionnement des bars du quartier festif voisin (Bairro Alto) ${ }^{14}$. Dans ce contexte, le réaménagement de la rue Rose est présenté comme une manière de développer les usages diurnes. Pourtant, elle attire de grandes foules le soir, entraînant une nouvelle dynamique commerciale : le nombre d'établissements nocturnes s'accroît d'une quinzaine à la fin des années 2000 pour atteindre 41 établissements en 2018 (Nofre et al., 2018).

La concentration et le développement des festivités nocturnes relèvent d'une dynamique complexe, faisant intervenir la capacité d'adaptation des établissements commerciaux, les politiques urbaines, les préférences des individus et leurs réseaux de sociabilité (Guillemot, Jaurand, 2018). Cependant, l'aménagement urbain joue un rôle central dans le choix des sortants (Guérin, 2017). À Montpellier, la piétonnisation est mise en avant par les sortants qui font de l'Écusson une destination de leurs sorties

I'Hérault. En ligne, consulté le 28 Décembre 2019. URL : https://www.adil34.org/fileadmin/Sites/ ADIL_34/etudes_observatoire_logement/Note_accession_16_version_def.pdf.

${ }^{11}$ Entretien avec un intervenant associatif, Montpellier, 2017.

12 Entretien avec la chargée de mission d'urbanisme et occupation du domaine public, Montpellier, 2017.

${ }^{13}$ Entretien avec un résident, Montpellier, 2016.

${ }^{14}$ Neves F., « Renovação do Cais do Sodré "corre o risco de falhar" », O Corvo, 12 septembre 2014. En ligne, consulté le 8 janvier 2020. URL : http://ocorvo.pt/2014/09/12/renovacao-do-cais-do-sodrecorre-o-risco-de-falhar/. 
nocturnes : « Avoir un centre-ville piéton, c'est très agréable. Tu peux te balader ou t'installer dans une terrasse sans le bruit des voitures ${ }^{15}$. »

Dans le même temps, la piétonnisation favorise l'installation des terrasses, qui sont une composante importante de l'attractivité et du modèle économique des établissements du centre-ville de Montpellier. Les terrasses permettent de doubler le nombre de places des établissements, souvent limitées à l'intérieur des établissements :

Nous fermons le bar la semaine prochaine... La terrasse est toujours pleine mais est trop petite et je n'arrive pas à avoir un chiffre d'affaires suffisant ${ }^{16}$.

À partir d'avril/mai, je reste toujours en terrasse. S'il n'y a pas de place, je vais voir dans un autre bar plutôt que m'installer à l'intérieur ${ }^{17}$.

$\mathrm{Au}$ Cais do Sodré, la piétonnisation de la rue a elle aussi produit de nouveaux usages nocturnes en extérieur, la rue étant occupée par des centaines de sortants tous les soirs $^{18}$. Comme dans d'autres villes (Melé, 2004 ; Comelli, 2016 ; Chausson, 2018), cette attractivité nocturne n'était pas anticipée et/ou souhaitée ni par les concepteurs, ni par les élus, mais a été progressivement acceptée par les gestionnaires de la ville. Elle est cohérente avec l'image que les deux villes veulent développer, à savoir une ville dynamique et jeune. Ainsi l'Office de tourisme de Montpellier a eu pour longtemps comme slogan: « La ville où le soleil ne se couche jamais » (figure ${ }^{\circ} 1$ ). Aujourd'hui, leur publicité présente la ville comme « fiesta friendly ${ }^{19}$ ».

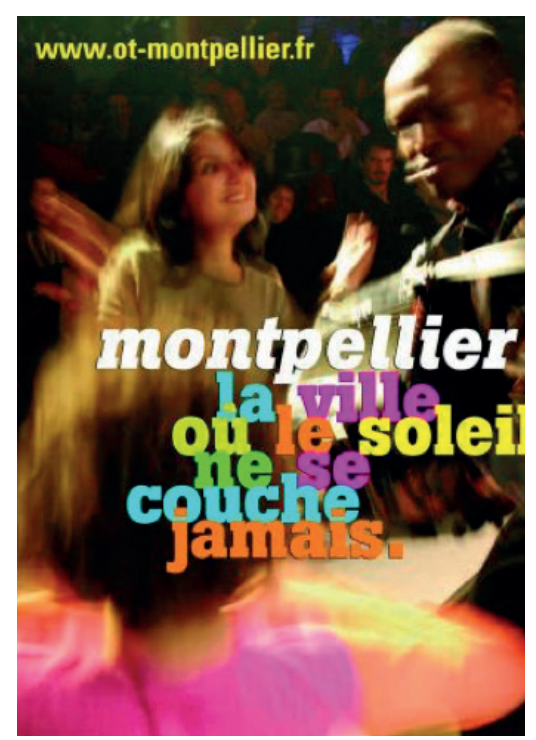

Figure nº 1 : Affiche promotionnelle (Source : Office de tourisme de Montpelier)

\footnotetext{
${ }^{15}$ Entretien avec un résident de 27 ans, Montpellier, février 2017.

${ }^{16}$ Entretien avec un gestionnaire d'établissement, Montpellier, décembre 2018.

17 Entretien avec un résident de 29 ans, Montpellier, septembre 2018.

18 Sobral C., « O Cais do Sodré mudou e os moradores não estão a gostar », Público, 4 juillet 2012. En ligne, consulté le 15 janvier 2020. URL: https://www.publico.pt/2012/04/07/local/noticia/o-caisdo-sodre-mudou-e-os-moradores-nao-estao-a-gostar-1541135.

${ }^{19}$ En ligne, consulté le 9 janvier 2020. URL : https://www.montpellier-tourisme.fr/Preparer-Reserver/ Vous-etes-plutot/Montpellier-fiesta-friendly.
} 
À Lisbonne, l'Office de tourisme vante les mérites de sa vie nocturne, et ce, jusqu'à l'aube : "The city has whole neighbourhoods converted to fun after dark and the spirit of bohemia. [...] Things start to get lively at around midnight with many clubs only opening their doors at around $2 \mathrm{am}$. And the fun is guaranteed all through the night until sunrise $^{20}$. » Ceci fait écho à la manière dont l'adjoint chargé de l'urbanisme à la mairie de Lisbonne présente sa politique comme répondant aux attentes des « nouvelles générations » pour une « ville vivante» :

Je crois qu'il y a plusieurs signes [...] et l'un des plus importants est le magazine Time Out ${ }^{21}$, à cause de ce qu'il révèle de l'énorme potentiel de Lisbonne. Tous les jours, il y a de nouvelles choses, des événements, de nouveaux magasins, de nouvelles activités [...]. Lisbonne est une ville vivante. II y a six ans, il n'y avait pas de kiosques, pas de terrasses... II y a, en effet, un changement très significatif22.

Cependant l'animation nocturne a aussi produit une intensification des conflits de voisinage, montrant une difficile conciliation avec les objectifs d'attractivité résidentielle. Comme le montrent plusieurs travaux, certains habitants perçoivent les usages festifs nocturnes comme dérangeants (Hae, 2011 ; Jeanmougin, 2019). À Montpellier, ces conflits s'articulent surtout autour du bruit produit par les pratiques nocturnes dans l'espace public (Louvet, 2010) : « Il y a eu un changement dans l'Écusson... qui coïncide avec la loi de ne pas fumer dans les lieux publics, ce qui a développé la présence de la population dans la rue, devant les bars et les restaurants pour fumer ${ }^{23}$. »

Ces usages sont aussi liés à la géographie des établissements nocturnes montpelliérains. Presque la totalité des établissements du centre-ville ferment à $1 \mathrm{~h}$ ( $2 \mathrm{~h}$ l'été), mais les discothèques étant principalement situées dans les communes autour de Montpellier, elles sont difficilement accessibles par une population étudiante non motorisée (Giordano et al., 2019) :

Le second problème [...], c'est la déambulation d'ivrognes de 16 à 70 ans, peu importe l'âge, qui circulent dans la rue Gagne Petit. Il y a un bar dans cette rue qui est fréquenté par une population de jeunes étudiants mais un peu marginale. Quand le bar ferme, les gens restent à la sortie et jouent du tam-tam... jusqu'à trois, quatre heures du matin ${ }^{24}$ !

\footnotetext{
${ }^{20}$ En ligne, consulté le 9 janvier 2020. URL : https://www.visitlisboa.com/en/c/eat-and-drink/barsand-nightlife.

21 Time Out est une publication internationale dédiée à la culture et aux styles de vie urbains, qui se décline en éditions localisées, dont Lisbonne. Dans cette ville, le magazine est également devenu acteur urbain, d'abord en tant que concessionnaire de kiosques, ensuite en tant que concessionnaire, puis propriétaire, d'un marché couvert - au Cais do Sodré - transformé en food court et lieu d'événements, dont une boîte de nuit.

22 Salgado M., « Entrevista Manuel Salgado, Vice-Presidente da CML: Lisboa é o cais da Europa, Turismo de Lisboa, juin 2013, n¹14, p. 16.

${ }^{23}$ Entretien avec un intervenant associatif, Montpellier, juin 2016.

${ }^{24}$ Entretien avec un résident, Montpellier, novembre 2016.
} 


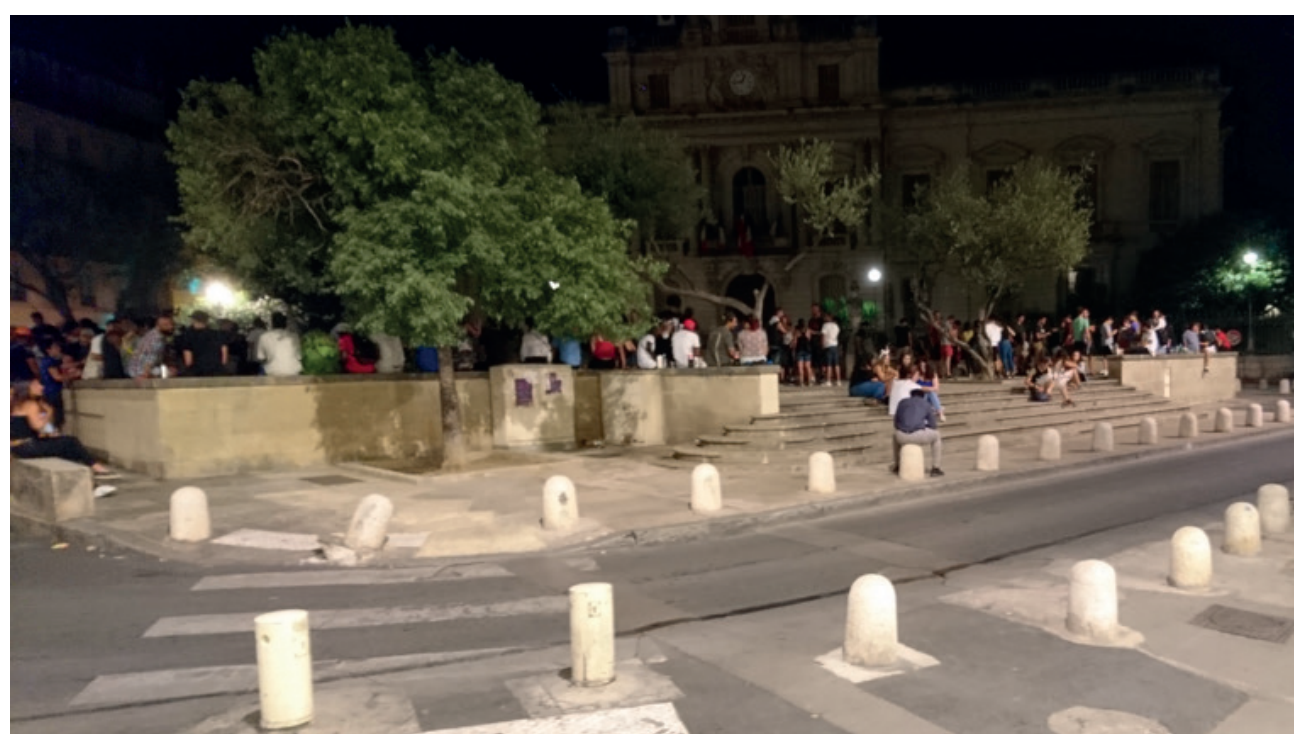

Figure nº 2 : Esplanade Léo Mallet, Montpellier (22 juillet 2018, 0 h 47)

(Source : Emanuele Giordano)

Au Cais do Sodré, des habitants créent une association, aujourd'hui nommée Aqui Mora Gente ("Y’a du monde qui habite ici »). Portée par des propriétaires ayant acheté depuis une quinzaine d'années dans le quartier ${ }^{25}$, l'association dénonce le bruit, la saleté et l'insécurité que ses membres associent aux festivités nocturnes et surtout aux pratiques de l'espace public, intimant la municipalité à une action plus poussée sur le sujet. Son répertoire d'action inclut la dénonciation en ligne, le dépôt de plaintes, l'interpellation d'acteurs publics (surtout municipaux) et la soumission de projets de communication et de sensibilisation au budget participatif municipal ${ }^{26}$.

Ainsi au cours des dernières années, les deux quartiers ont connu des conflits entre les habitants qui ont investi dans la constitution d'un quartier résidentiel et les sortants. Les riverains mettent non seulement en avant des arguments juridiques, mais dénoncent aussi la contradiction entre ce qu'ils perçoivent comme une excessive tolérance vis-à-vis des sortants nocturnes, l’objectif affiché de réhabiliter le centre-ville et le risque d'une polarisation sociale du centre-ville :

À cause d'une vie assez tendue en centre-ville et les bruits qui sont liés, les personnes quittent le centre-ville et cette population est remplacée par des étudiants qui se mettent en colocation ${ }^{27}$.

Il faut savoir que la multiplication des départs n'est pas explicite, mais il y a eu un départ progressif des populations plus âgées... II faut faire attention au risque de « ghettoïser » le centre-ville avec des « pauvres » et des étudiants ${ }^{28}$.

\footnotetext{
${ }^{25}$ Alemão S., "Cais do Sodré tem assistido ao regresso do mau ambiente e da insalubridade nos últimos meses », O Corvo, 30 octobre 2018. En ligne, consulé le 8 janvier 2020. URL : https://ocorvo.pt/ cais-do-sodre-tem-assistido-ao-regresso-do-mau-ambiente-e-da-degradacao-nos-ultimos-meses/. ${ }^{26}$ Voir le site de l'association en ligne, consulté le 9 janvier 2020. URL : https://aquimoragente. weebly.com/.

27 Entretien avec un résident, Montpellier, septembre 2016.

${ }^{28}$ Entretien avec un intervenant associatif, Montpellier, 2016.
} 
Dans les deux cas, l'intensification des pratiques de loisir nocturnes apparaît soit inattendue, soit sous-estimée par les acteurs publics dans ce qu'elle implique de contradictoire avec l'attractivité résidentielle.

\section{Les écueils de l'intégration de la vie nocturne dans les pratiques d'urbanisme}

Face à l'émergence de ces utilisations simultanées, mais antagonistes, d'un même espace (Mallet, Burger, 2016), les réponses politiques sont variées : création de campagnes de sensibilisation et de médiation (de Maillard, 2013), mais aussi approches disciplinaires qui envisagent les effets produits par les pratiques récréatives nocturnes à travers le prisme de l'illégalité ou de l'excès (Nofre, Eldridge, 2018). La multiplicité d'approches relève, au moins partiellement, des différents intérêts qui animent les acteurs qui participent à la régulation des activités festives nocturnes (Walker, 2017). Elle traduit aussi une hiérarchisation des activités nocturnes selon la perception de leur valeur économique et symbolique et, ce faisant, des différents publics sortants. Encore une fois, les cas de Lisbonne et Montpellier, où les deux mairies ont renforcé la réglementation de l'usage nocturne de l'espace public - la fermeture des parcs publics et des établissements festifs la nuit -, illustrent bien cette complexité.

À Lisbonne, la municipalité défend sa posture réactive - c'est-à-dire la gestion des conflits découlant des aménagements de l'espace public, plutôt que leur anticipation en affirmant l'importance des effets de mode dans les lieux des pratiques festives nocturnes ${ }^{29}$. Il serait ainsi peu judicieux de trop réguler ces activités en amont, voire de les intégrer aux pratiques de conception des espaces. Cette posture peut partiellement expliquer l'absence de précautions sur les éventuelles nuisances lors de la piétonnisation de la rue Rose en 2011 et de sa reconduction en 2013, et ce malgré l'opposition des riverains.

Cette logique d'action réactive se traduit dans la construction progressive d'un dispositif de régulation de la nuit au Cais do Sodré, tout en nourrissant des réflexions à l'échelle du centre historique, voire de la commune. Un premier volet d'intervention municipale porte sur les établissements ouverts la nuit. Des despachos (équivalents à des arrêtés municipaux) sont publiés et visent soit le quartier, soit des périmètres plus larges. Ainsi, ils accompagnent le déplacement des pratiques festives nocturnes et/ou l'intensification des conflits de voisinage dans le territoire municipal. Ces mesures (cf. tableau $n^{\circ}$ 1) raccourcissent progressivement les horaires de fonctionnement des établissements, différenciant les types d'établissements, de manière à diminuer la durée des usages festifs nocturnes. Elles sont par ailleurs accompagnées d'un durcissement des pénalités pour les infractions des établissements en matière de bruit et d’une augmentation des moyens d'inspection.

\footnotetext{
${ }^{29}$ Moura Ramos A., Cepa P., Caos do Sodré [Reportage], 22 octobre 2014. En ligne, consulté le 8 jan-
} vier 2020. URL : https://www.youtube.com/watch?v=0GIGaO9IeS4. 


\begin{tabular}{|c|c|c|c|}
\hline Année & Mesure & Périmètre & Source \\
\hline 2012 & $\begin{array}{l}\text { Interdiction des after } \\
\text { hours }\end{array}$ & Cais do Sodré & Despacho 100/P/2012 \\
\hline 2012 & $\begin{array}{l}\text { «Permis zéro »: } \\
\text { suppression des } \\
\text { démarches } \\
\text { d'ouverture } \\
\text { d'établissements }\end{array}$ & National & Decreto-Lei 48/2011 \\
\hline 2014 & $\begin{array}{l}\text { Harmonisation des } \\
\text { horaires avec Bairro } \\
\text { Alto : } 22 \text { h pour } \\
\text { épiceries de nuit, } \\
2 \mathrm{~h} / 3 \text { h pour les bars } \\
\text { et restaurants, } 4 \mathrm{~h} \\
\text { pour bars avec piste } \\
\text { de danse }\end{array}$ & $\begin{array}{l}\text { Cais do Sodré, Bica et } \\
\text { Santos }\end{array}$ & Despacho 140/P/2014 \\
\hline 2014 & $\begin{array}{l}\text { Interdiction de } \\
\text { vente de boissons } \\
\text { vers l'extérieur à } \\
\text { partir de } 1 \mathrm{~h}\end{array}$ & $\begin{array}{l}\text { Cais do Sodré, Bica et } \\
\text { Santos }\end{array}$ & Despacho 140/P/2014 \\
\hline 2015 & $\begin{array}{l}\text { Libéralisation des } \\
\text { horaires de } \\
\text { fonctionnement des } \\
\text { établissements com- } \\
\text { merciaux }\end{array}$ & National & Decreto-Lei nº 10/2015 \\
\hline 2016 & $\begin{array}{l}\text { Horaires s'appli- } \\
\text { quant à toute la } \\
\text { ville, sauf pour zone } \\
\text { non résidentielle au } \\
\text { bord du Tage, sans } \\
\text { restriction d'horaire }\end{array}$ & $\begin{array}{l}\text { Commune de } \\
\text { Lisbonne }\end{array}$ & Aviso $\mathrm{n}^{\circ} 13367 / 2016$ \\
\hline 2016 & $\begin{array}{l}\text { Obligation de } \\
\text { capteurs de son, } \\
\text { contrôlés en temps } \\
\text { réel ; d'insonoriser } \\
\text { et de fermer portes } \\
\text { et fenêtres }\end{array}$ & $\begin{array}{l}\text { Commune de } \\
\text { Lisbonne }\end{array}$ & Aviso $\mathrm{n}^{\circ} 13367 / 2016$ \\
\hline
\end{tabular}

Par ailleurs, les mesures prises par la municipalité reconnaissent l'existence d'un « effet de réseau ${ }^{30}$ » (Despacho 100/P/2012) dans les pratiques nocturnes, selon lequel la

30 Pourtant, cet effet de réseau ne semble être pris en compte qu'à des échelles plus larges, vu la persistance de décisions sur les horaires de fonctionnement d'établissements ou d'ouverture d'espaces publics qui n'anticipent pas le déplacement des « nuisances » qu'elles visent à limiter. Cf. Alemão S., "Cais do Sodré tem assistido ao regresso do mau ambiente e da insalubridade nos últimos meses », O Corvo, 30 octobre 2018. En ligne, consulté le 8 janvier 2020. URL : https://ocorvo.pt/ 
concentration d'établissements attire de nombreux consommateurs et encourage des pratiques sur la voie publique - jusqu'à définir la consommation d'alcool sur la voie publique comme le principal problème en matière de nuisances ${ }^{31}$. Ainsi, les mesures de la municipalité cherchent à contrôler la consommation d'alcool sur la voie publique, en interdisant, dans le Despacho 140/P/2014, la vente vers l'extérieur à partir de $1 \mathrm{~h}$, pour les bars et restaurants, et instituant la fermeture des épiceries de nuit à $22 \mathrm{~h}$.

Ce faisant, la municipalité fait écho aux arguments de certains commerçants, qui opposent leur offre et leur investissement dans la réhabilitation du quartier : «À Lisbonne, avec ce temps merveilleux, c'est génial de boire un verre dehors, mais il faut que ça se termine à une heure donnée. [Pour que ce soit compatible avec les usages résidentiels] et pour des questions basiques de concurrence [...], ces bars n'offrent rien d'autre que des boissons moins chères qu'ailleurs ${ }^{32}$."

L'efficacité de l'action municipale est également mise à l'épreuve par des lois nationales qui suppriment les démarches administratives d'octroi de permis à des établissements de restauration et de débit de boissons en 2012 et libéralisent les horaires de fonctionnement des établissements commerciaux en 2015. Si la municipalité demeure compétente pour définir les horaires et les secteurs interdits à certains types d'établissements en aval ${ }^{33}$, cette libéralisation concerne des établissements vendant de l'alcool destiné à être consommé à l'extérieur.

Un deuxième volet d'intervention est la mise en place de dispositifs de concertation, d'abord à l'échelle du quartier puis à celle de la municipalité, et l'organisation de projets partenariaux par les résidents, les commerçants et le secteur associatif et universitaire visant à la mise en réseau et la capacitation des acteurs de la vie nocturne aux enjeux de santé publique et de sûreté. L'efficacité de ces instances à diminuer les nuisances a été critiquée par les participants et chercheurs ${ }^{34}$, malgré leur capacité à trouver des compromis contribuant aux règlements municipaux. Ces démarches montrent par ailleurs la reconnaissance de la légitimité des différents intérêts en jeu et de la difficulté de les concilier. L'inefficacité perçue de ces instances découle d'une difficile et insuffisante mise en œuvre des mesures. Malgré les moyens de contrôle accrus ${ }^{35}$, les pratiques nocturnes en espace public restent difficiles à réguler.

cais-do-sodre-tem-assistido-ao-regresso-do-mau-ambiente-e-da-degradacao-nos-ultimos-meses/. 31 Soares M., « Bares no Cais do Sodré vão encerrar mais cedo mas álcool na rua mantém-se », Público, 23 octobre 2014. En ligne, consulté le 3 novembre 2014. URL: https://www.publico. pt/2014/10/23/local/noticia/bares-do-cais-do-sodre-vao-encerrar-mais-cedo-mas-donos-tememque-fechem-de-vez-1673787.

32 Entretien avec un commerçant, Lisbonne, 10 septembre 2013. Traduction personnelle.

${ }^{33}$ Filipe C., «Bairro Alto interdito a novos bares, o que é uma vitória para os moradores », Público, 30 mai 2012. En ligne, consulté le 8 janvier 2020. URL : https://www.publico.pt/2012/05/30/local/ noticia/bairro-alto-interdito-a-novos-bares-o-que-e-uma-vitoria-para-os-moradores-1548162.

34 Moura Ramos A., Cepa P., Caos do Sodré [Reportage], 22 octobre 2014. En ligne, consulté le 8 janvier 2020. URL : https://www.youtube.com/watch?v=0GIGaO9leS4 ; Nofre et al. (2018).

35 "Câmara de Lisboa mandou encerrar seis espaços de diversão noturna em dois anos », TVI24, 13 décembre 2017. En ligne, consulté le 8 janvier 2020. URL: https://tvi24.iol.pt/sociedade/discotecas-fechadas/camara-de-lisboa-mandou-encerrar-seis-espacos-de-diversao-noturna-em-dois-anos; Moura Ramos A., Cepa P., Caos do Sodré [Reportage], 22 octobre 2014. En ligne, 
À Montpellier, les conflits entre sortants et résidents en centre-ville ne sont pas récents (Louvet, 2010) et sont le plus souvent liés à la consommation d'alcool. Comme à Lisbonne, cela a entraîné une hiérarchisation entre les différents établissements nocturnes. Plusieurs arrêtés municipaux sont ainsi mis en place depuis 2005, interdisant la consommation d'alcool dans l'espace public en dehors de terrasses et limitant l'activité des épiceries : interdiction de vendre de l'alcool après $22 \mathrm{~h}$, fermeture obligatoire entre $2 \mathrm{~h}$ et $6 \mathrm{~h}$ dans plusieurs quartiers y compris le centre-ville pendant l'été (tableau n 2 ).

Pour mieux vérifier l'application de cette réglementation, la municipalité a créé en 2015 une brigade de nuit au sein de la police municipale. La création de cette brigade s'est accompagnée d'un contrôle plus rigoureux de la fermeture des parcs publics (Giordano et al, 2019).

Cependant, en dépit de ces mesures, l'attitude de la municipalité envers l'activité des établissements nocturnes n'est pas dépourvue d'ambiguïté : dérogation des horaires de fonctionnement en été (de $1 \mathrm{~h}$ à $2 \mathrm{~h}$ ), réduction des prix des permis d'occupation de l'espace public pour les terrasses dans certaines rues du centre-ville ${ }^{36}$ et mesures pour soutenir l'activité économique des établissements.

\begin{tabular}{|l|l|l|l|}
\hline Année & Mesure & Périmètre & Source \\
\hline 2005 & $\begin{array}{l}\text { Interdiction vente } \\
\text { de boissons } \\
\text { alcoolisées à } \\
\text { importer à partir de } \\
22 \mathrm{~h}\end{array}$ & $\begin{array}{l}\text { Commune de } \\
\text { Montpellier }\end{array}$ & $\begin{array}{l}\text { Arrêté Hygiène et } \\
\text { Santé 5/11 }\end{array}$ \\
\hline 2012 & $\begin{array}{l}\text { Fermeture épiceries } \\
\text { de nuit entre 2 } \mathrm{h} \text { et } \\
6 \mathrm{~h} \text { du matin }\end{array}$ & $\begin{array}{l}\text { Écusson et trois } \\
\text { autres secteurs de } \\
\text { la ville }\end{array}$ & Arrêté $\mathrm{n}^{\circ}$ 2012/1299 \\
\hline 2016 & $\begin{array}{l}\text { Fermeture des } \\
\text { débits de boissons à } \\
1 \mathrm{~h}\end{array}$ & $\begin{array}{l}\text { Préfecture de } \\
\text { l'Hérault }\end{array}$ & $\begin{array}{l}\text { Arrêté } \\
\mathrm{n}^{\circ} \text { 2016-I-DEB-I }\end{array}$ \\
\hline 2016 & $\begin{array}{l}\text { Interdiction de la } \\
\text { consommation } \\
\text { alcool en dehors des } \\
\text { terrasses }\end{array}$ & $\begin{array}{l}\text { Écusson et trois } \\
\text { autres secteurs de } \\
\text { la ville }\end{array}$ & Arrêté $\mathrm{n}^{\circ}$ 2016/1107 \\
\hline
\end{tabular}

Ces mesures n’ont pas satisfait les résidents mobilisés : « Mr Saurel [le Maire] exprime l'idée que "si on ne veut pas de bruit, il ne faut pas habiter dans l'Écusson". Il a une politique et une volonté de ne pas être en opposition avec l'installation de bars et autres lieux de nuit ${ }^{37}$.»

L'enquête à Montpellier fait par ailleurs ressortir que les nuisances perçues ont une dimension structurelle liée à la configuration du centre-ville : le climat favorable, la

consulté le 8 janvier 2020. URL : https://www.youtube.com/watch?v=0GIGaO9leS4.

36 Pour une terrasse simple dépourvue d'équipements, le prix est passé de 97 à $60 € \mathrm{~m}^{2} / \mathrm{an}$. Entretien avec le Service occupation sol public de la ville de Montpellier, juin 2017.

${ }^{37}$ Entretien avec un intervenant associatif, Montpellier, 2017. 
piétonnisation et la concentration d'établissements et terrasses font que, à tout moment, le nombre d'usagers dans l'espace public la nuit est élevé. Ces activités, même si elles ne sont pas caractérisées par la consommation d'alcool ou que leur intensité sonore n'est pas élevée, continuent à susciter la gêne chez certains résidents :

Parce que la configuration du centre-ville de Montpellier est assez particulière. Les rues sont étroites, il y a un effet canyon, les bruits sont forts, ça résonne. Par exemple, je connais une personne qui vit à l'esplanade de l'Europe, c'est plus ouvert, plus étendu ${ }^{38}$.

La spécificité montpelliéraine d'avoir un centre piéton accentue ces nuisances ou du moins les représentations qu'on en a. En effet, la piétonnisation accentue la focalisation sur les nuisances notamment sonores. S'il y avait des voitures en centre-ville, les problèmes seraient différents ${ }^{39}$.

Ces complexités soulignent non seulement l'importance de mettre en place des formes de gouvernance de l'espace-temps nocturne qui permettent la coordination et la concertation entre les acteurs publics et privés (Guérin, 2019), mais aussi l'intérêt d'intégrer à ces démarches les acteurs de l'urbanisme. À l'heure actuelle, l'articulation des loisirs nocturnes aux pratiques urbanistiques est un des angles morts des politiques de revitalisation des quartiers historiques des villes européennes. Elle met en évidence la complexité de concilier, dans un même espace, l'attractivité résidentielle, touristique et les loisirs nocturnes. Certains des effets produits par la vie nocturne (bruit, déchets, etc.) sont difficilement compatibles avec le cadre de vie recherché par les gentrifieurs (Jeanmougin, 2019), d'autant plus que ces conséquences sont souvent exacerbées par la morphologie des quartiers historiques privilégiés par ces opérations de revitalisation (Roberts, Eldridge, 2009). Les études disponibles sur l'évolution des polarités nocturnes semblent indiquer deux trajectoires de développement majeures : la progressive expulsion des activités nocturnes à la suite d'un durcissement de la réglementation qui répond aux demandes des nouveaux résidents (Hae, 2011 ; Comelli, 2016 ; Jeanmougin, 2019) ; ou une spécialisation vers une population résidente, en termes de tranches d'âge, de styles de vie relativement synchronisés aux rythmes des loisirs nocturnes (étudiants, touristes, etc.) à laquelle se rajoutent parfois des populations moins aisées pour lesquelles les polarités nocturnes, en raison des prix des loyers inférieurs à la moyenne, représentent un des seuls accès aux centres-villes (Nofre et al., 2018). Dans ce contexte, l'anticipation de ces trajectoires et leur prise en compte dans les opérations d'aménagement peuvent permettre une gestion plus efficace des conflits temporels, tensions générées par des usages simultanés et antagonistes d'un lieu. Ainsi, de nouvelles actions commencent à voir le jour. À Barcelone, par exemple, dans un secteur prochainement piétonnisé, la ville a créé une zone protégée en matière de débits de boissons. Cette prise en compte $a$ priori des potentiels impacts économiques et temporels des opérations de piétonnisation peut limiter le développement des conflits

\footnotetext{
${ }^{38}$ Entretien avec un résident, mai 2018.

39 Entretien avec le Chargé de mission, Service communal d'hygiène et de santé, Montpellier, juin 2017.
} 
d'usages pendant les heures nocturnes et éviter des processus de gentrification commerciale (Özdemir, Selçuk, 2017). D’autres villes développent des réflexions sur la possibilité de concentrer l'offre nocturne dans des zones non résidentielles, comme à Lisbonne, tandis que Genève développe une offre résidentielle tournée vers les étudiants dans les polarités nocturnes (Pieroni, 2017). Ces nouvelles pratiques semblent indiquer une prise en compte des loisirs nocturnes dans les pratiques urbanistiques. Le choix semble davantage porter sur la séparation entre fonction résidentielle et loisirs nocturnes. Cependant, l'efficacité de ces démarches reste à évaluer. Parallèlement, elles posent d'emblée des questions d'ordre plus général. D’une part, elles risquent d'entériner les inégalités d'accès à la ville (différence d'accessibilité des centralités nocturnes périphériques, concentration de populations moins aisées dans des centralités de loisirs nocturnes). D'autre part, elles risquent de reproduire une spécialisation fonctionnelle (résidentielle, certains types de commerce, etc.) de certains morceaux des villes, qui, pour certains aménageurs, équivaudrait à créer des quartiers peu animés, voire « chiants ${ }^{40} »$.

\section{Conclusion}

Dans cet article, nous avons exploré les liens entre la production de la ville, notamment des espaces publics, et les conflits temporels émergeant de la cohabitation entre loisirs nocturnes et fonctions résidentielles. Pour ce faire, nous nous sommes appuyés sur deux villes : Montpellier et Lisbonne.

Nous avons d'abord montré la manière dont les usages nocturnes demeurent largement impensés dans la production des espaces publics. Alors même que la NTE est devenue un enjeu important, les pratiques d'urbanisme continuent d'ignorer leurs éventuels impacts en matière de partage temporel de la ville et des conflits qui peuvent en découler.

Conséquence inattendue de pratiques d'urbanisme convivial qui « réussissent », les loisirs nocturnes deviennent alors un objet complexe de régulation publique. Nous avons exploré cette complexité dans les deux villes, devant à la fois concilier les intérêts légitimes des différents acteurs - dont les sortants eux-mêmes - et se confronter aux différentes dimensions des loisirs nocturnes à l'origine des conflits. Les démarches de gestion des loisirs nocturnes les plus répandues sont réactives et impliquent des mesures répressives par les municipalités, bien que ces instances de gouvernance se révèlent souvent peu efficaces, comme le montre la permanence des conflits entre résidents et sortants.

Et pourtant, l'article montre en creux l'importance de l'intégration des acteurs de la production urbaine dans la gestion et la régulation des pratiques festives nocturnes, y compris en amont de la conception des projets de réhabilitation des centres-villes.

${ }^{40}$ Les Entretiens de l'aménagement, grande rencontre organisée périodiquement par les aménageurs français réunis au club Ville et Aménagement, avaient en 2019 la «ville pas chiante » comme un des thèmes de réflexion majeurs. 
Des efforts dans ce sens existent, mais comportent eux aussi des risques : production d'un simple déplacement des pratiques festives qu'ils cherchent à maîtriser, création de quartiers peu animés ne répondant pas aux attentes de citadins et acteurs de la production de la ville.

La nuit met alors en lumière certaines contradictions de l'urbanisme convivial, dont la plus flagrante est la conciliation de l'attractivité résidentielle, les pratiques de loisirs et la garantie d'un espace public accessible à d'autres usages que ceux proposés par l'offre marchande. Les résultats des tentatives de prise en compte des usages festifs nocturnes en amont des opérations d'aménagement doivent ainsi être analysés par le prisme de la gestion des conflits et de leurs conséquences sur les caractéristiques sociodémographiques des populations résidentes.

\section{Bibliographie}

Bianchini F. (1995), « Night Cultures, Night Economies », Planning Practice er Research, vol. 10, n², p. 121-26.

Brennac T., Reighner H., Hernandez F. (2013), « Centres-villes aménagés pour les piétons : développement durable ou marketing urbain et tri social ? ", Recherche Transports Sécurité, vol. 4, p. 267-278.

CAMARINHAS C. (2011), L'urbanisme de Lisbonne. Éléments de théorie urbaine appliquée, Paris, L'Harmattan.

Chatterton P., Hollands R. (2003),Urban Nightscapes: Youth Cultures, Pleasure Spaces and Corporate Power, London, Routledge.

Chausson N. (2018), " Prendre en compte les usages nocturnes dans les projets d'aménagements urbains. L'exemple des berges du Rhône à Lyon », in F. Guérin, E. Hernandez-Gonzalez, A. Montadon, Cohabiter les nuits urbaines : des significations de l'ombre aux régulations de l'investissement ordinaire des nuits, Paris, L'Harmattan, p. 114-128.

Clerval A., Fleury A. (2009), « Politiques urbaines et gentrification, une analyse critique à partir du cas de Paris », L'Espace politique. En ligne, consulté le 12 avril 2019. URL : https://journals.openedition.org/espacepolitique/1314\#quotation.

Comelli C. (2016), « Politique de revitalisation et nuits urbaines : le cas de Bordeaux », L'Espace politique. En ligne, consulté le 12 avril 2019. URL : https://journals. openedition.org/espacepolitique/3988\#quotation.

Elias N., Dunning E. (1986), Quest for Excitement: Sport and Leisure in the Civilizing Process, London, Blackwell Publishers.

FERRAS R. (1978), Écusson et Polygone : enfants et retraités dans le centre de Montpellier, Montpellier, Société languedocienne de géographie. 
FleURY A. (2007), Les espaces publics dans les politiques métropolitaines. Réflexions au croisement de trois expériences : de Paris aux quartiers centraux de Berlin et Istanbul, thèse de doctorat, Paris, Université de Paris 1 Panthéon-Sorbonne.

Geoffray M.-L., Le Renard A., Laplanche-Servigne S. (2012), « Comparer a posteriori : retour sur une expérience collective de recherche », Terrains e Travaux, n² 21, p. 165-180.

GiBand D. (2017) « "Creative Urbanism” in the French South: Constructing the (Unequal) Creative City in Montpellier », in U. Gerhard, M. Hoelscher, D. Wilson, Inequalities in Creative Cities, London, Palgrave Macmillan, p. 165-185.

Giordano E., Manella G., Rimondini T., Crozat D. (2019), «The Spatio-temporal Geographies of Public Spaces at Night and their Regulation as Source of Conflict. The Cases of Montpellier and Bologna », Espace, Populations, Sociétés. En ligne, consulté le 11 janvier 2020. URL : http://journals.openedition.org/eps/8725.

Gomes P. (2017), La production de l'espace public à Lyon, Lisbonne et Louvain-la-Neuve : politiques, processus et prise en compte des usages, thèse de doctorat, Paris, Université Paris-Est.

Gomes P. (2020), « The Birth of Public Space Privatization: How Entrepreneurialism, Convivial Urbanism and Stakeholder Interactions made the Martim Moniz Square, in Lisbon, 'Privatization-ready' », European Urban and Regional Studies, vol. 27, n 1 , p. 86-100.

GuÉrin F. (2017), Enjeux socio-urbains du noctambulisme : les cas de Paris et Madrid au début $d u$ XXIe siècle, thèse de doctorat en aménagement, Paris, Université Paris-Est.

GuÉRIN F. (2019), « Quel(s) droit(s) aux nuits festives parisiennes ? Analyse critique de la gouvernance de la vie nocturne », Métropolitiques. En ligne, consulté le 12 avril 2019. URL:https://www.metropolitiques.eu/Quel-s-droit-s-aux-nuits-festives-parisiennes. html.

Guillemot L., JauRand E. (2018), « La fièvre du jeudi soir : soirées festives étudiantes et commerce de centre-ville à Angers », Bulletin de l'association de géographes français, vol. $95 n^{\circ} 1$, p. 97-112.

Hadfield P., Lister S., Hobbs D., Winlow S. (2001), « The '24-hour city' - condition critical », Town and Country Planning, vol. 70, $\mathrm{n}^{\circ}$ 11, p. 300-302.

HAE L. (2011), «Gentrification and Politicization of Nightlife in New York city », ACME, vol. 11, $n^{\circ} 3$, p. 564-584.

HaRvey D. (1989), « From Managerialism to Entrepreneurialism: The Transformation in Urban Governance in Late Capitalism », Geografiska Annaler. Series B, Human Geography, vol. 71, n 1, p. 3-17.

Jeanmougin H. (2019), "Vie nocturne animée : moteur ou frein à la gentrification ? Conflit d'usage et enjeux contradictoires dans le quartier de la Magione à Palerme », Bollettino della Società Geografica Italiana, vol. 14, nº 1, p. 231-240. 
LestegÀs I. (2019), " Lisbon After the Crisis: From Credit fuelled Suburbanization to Tourist-driven Gentrification ", International Journal of Urban and Regional Research, vol. 43, n 4, p. 705-723.

Louvet P. (2010), « La problématique du bruit à Montpellier : acteurs, gestions et représentations », mémoire de master 2 en aménagement, Montpellier, Université Paul Valéry Montpellier 3.

Maillard J. de (2013), «Réguler les espaces publics : le rôle ambivalent des nouveaux métiers », Métropolitiques. En ligne, consulté le 11 janvier 2019. URL : https://www. metropolitiques.eu/Reguler-les-espaces-publics-le.html.

Malheiros J., Carvalho R., Mendes L. (2012), « Etnicização residencial e nobilitação urbana marginal: processo de ajustamento ou prática emancipatória num bairro do centro histórico de Lisboa? ", Sociologia, Revista da Faculdade de Letras da Universidade do Porto, Número temático: Imigração, Diversidade e Convivência Cultural, p. 97-128.

Mallet S. (2013), "Aménager les rythmes : politiques temporelles et urbanisme ", Espacetemps.net. En ligne, consulté le 12 avril 2019. URL : https://www.espacestemps. net/en/articles/amenager-les-rythmes-politiques-temporelles-et-urbanisme/.

Mallet S., Burger C. (2016), « What place for night-time in the urban policy of a French intermediate city? ", Articulo. En ligne, consulté le 12 avril 2019. URL : https:// journals.openedition.org/articulo/3042.

Mele P. (2004), « Habitants mobilisés et devenir d’un espace patrimonial ? „ Géocarrefour, vol. 79, n 3, p. 223-230.

Nofre J. (2013), « “Vintage Nightlife”: Gentrifying Lisbon downtown », Fennia, vol. 191, $\mathrm{n}^{\circ} 2$, p. 106-121.

Nofre J., EldRidge A. (2018), Exploring Nightlife: Space, Society and Governance, London, Rowman \& Littlefield International.

Nofre J., Martins J. C., Vaz D., Fina R., Sequera J., Vale P. (2018), « The 'Pink Street' in Cais do Sodré: Urban Change and Liminal Governance in a Nightlife District of Lisbon », Urban Research er Practice, vol. 12, $\mathrm{n}^{\circ}$ 4, p. 322-340.

Özdemir D., SelçuK I. (2017), « From Pedestrianisation to Commercial Gentrification: The case of Kadıköy in Istanbul », Cities, vol. 65, p. 10-23.

Petonnet C. (1982), "L'Observation flottante : L'exemple d'un cimetière parisien », L'Homme : études d'anthropologie urbaine, vol. 22, $\mathrm{n}^{\circ}$ 4, p. 37-47.

PIERONI R. (2017), Institutionnaliser la nuit : géographie des politiques nocturnes à Genève, thèse de doctorat, Genève, Université de Genève.

Roberts M., Eldridge A. (2009), Planning the Night-Time City, London, Routledge.

Roberts M., Turner C., Greefield S., Osborne G. (2006), « A Continental Ambience? Lessons in Managing Alcohol-related Evening and Night-time Entertainment from four European Capitals », Urban Studies, vol. 43, n 7, p. 1105-1125. 
Rousseau M. (2009), « Re-imaging the City Centre for the Middle Classes: Regeneration, Gentrification and Symbolic Policies in "Loser Cities" », International Journal of Urban and Regional Research, vol. 33, $n^{\circ}$ 3, p. 770-788.

Santos J. R. (2019), « Public Space, Tourism and Mobility », The Journal of Public Space. En ligne, consulté le 8 janvier 2020. URL : https://doi.org/10.32891/jps.v4i2.1203.

SHAw R. (2015), « Night as Fragmenting Frontier: Understanding the Night That Remains in an Era of 24/7 », Geography Compass, vol. 9, n 12, p. 637-47.

VAN LIEMPT I., VAN AALST I., SchwANEN T. (2015), « Introduction: Geographies of the Urban Night », Urban Studies, vol. 52, n 3, p. 407-421.

Volle J.-P., Viala L., Négrier E., Bernie-Boissard C. (2010), Montpellier, la ville inventée, Marseille, Parenthèses.

WALKER É. (2017), « De la discipline au travail électoral ? Gouverner l'espace-temps récréatif nocturne à Rennes », Cultures efConflits, vol. 105-106, p. 123-43.

WALKER É. (2018), Quand la ville ne dort pas : s'approprier l'espace-temps hypercentral nocturne par et autour de l'usage récréatif. Les exemples de Caen et Rennes, thèse de doctorat, Caen, Université de Caen.

Wolifson P., DrozDZEwski D. (2017), « Co-opting the Night: The Entrepreneurial Shift and Economic Imperative in NTE Planning », Urban Policy and Research, vol. 35, $\mathrm{n}^{\circ} 4$, p. 486-504. 